\title{
The Asymptotics of Neutral Curve Crossing in Taylor-Dean Flow
}

C. P. Hills

Technological University Dublin, chris.hills@dit.ie

A. P. Bassom

University of Western Australia

Follow this and additional works at: https://arrow.tudublin.ie/scschmatart

Part of the Applied Mathematics Commons, Fluid Dynamics Commons, and the Mathematics

Commons

\section{Recommended Citation}

Hills, C.P. \& Bassom, A.P. (2006). The asymptotics of neutral curve crossing in Taylor-Dean flow, Zamp:Zeitschrift für Angewandte Mathematik und Physik (ZAMP). vol 57, no 1, pg. 76-93. doi:10.21427/ gzdh-4595

This Article is brought to you for free and open access by the School of Mathematics at ARROW@TU Dublin. It has been accepted for inclusion in Articles by an authorized administrator of ARROW@TU Dublin. For more information, please contact arrow.admin@tudublin.ie, aisling.coyne@tudublin.ie,gerard.connolly@tudublin.ie.

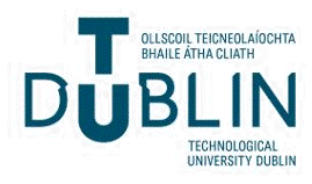




\title{
The asymptotics of neutral curve crossing in Taylor-Dean flow
}

\author{
C. P. Hills and Andrew P. Bassom
}

\begin{abstract}
The fluid flow between a pair of coaxial circular cylinders generated by the uniform rotation of the inner cylinder and an azimuthal pressure gradient is susceptible to both Taylor and Dean type instabilities. The flow can be characterised by two parameters: a measure of the relative magnitude of the rotation and pressure effects and a non-dimensional Taylor number. This work considers the small gap, large wavenumber limit for linear perturbations when the onset of the Taylor and Dean instabilities is concurrent. A consistent, matched asymptotic solution is found across the whole annular domain and identifies five regions of interest: two boundary adjustment regions and three internal critical points. Necessary conditions for the Taylor number and wavenumber are found and interpreted with reference to the suggestion of neutral curve kinks occurring at moderate wavelengths.
\end{abstract}

Mathematics Subject Classification (2000). 76E05.

Keywords. Stability, vortices, mode-crossing, matched asymptotics, concentric cylinder flow.

\section{Introduction}

The flow of a viscous fluid contained between two coaxial circular cylinders has been widely studied and its geometrical simplicity has ensured that Couette flow has become a paradigm for the study of a plethora of flow phenomena. The addition of an azimuthal pressure gradient, although difficult to realise in practice, provides an excellent model for curved channel flow and this composite TaylorDean problem has been discussed extensively in the literature; see, for example, [1]. In the limit of a small annular gap the unperturbed azimuthal flow is well known and the linear eigenvalue equations governing perturbations at neutral stability have been studied by, among others, Di Prima [2], Hall [3] and Kachoyan [4]. The eigenvalue problem is determined by three parameters: the axial wavenumber $k$ of the perturbations; $\beta$, a measure of the relative dominance of the effects of rotation and pressure and a Taylor number $\widehat{T}$. The value $\beta=0$ corresponds to Taylor-Couette flow and $\beta=1$ to classical Dean flow. For each value of $\beta$ there exists a critical Taylor number $\widehat{T}_{c}$; for $\widehat{T}>\widehat{T}_{c}$ the nature of the instability depends 
upon the values of $\beta$ and $k$. When the principal destabilising effect is rotation the instability will be akin to an isolated Taylor instability close to the inner cylinder. Conversely, when the main destabilising effect is pressure, a Dean-like instability will be observed in the main flow and away from the boundaries. At sufficiently large $\beta$ there exists a range of wavenumbers for which linear perturbations of neither pure Taylor nor Dean type exist but rather time-periodic solutions are the dominant instability. Chen \& Chang [5] conducted a numerical study of the onset of linear instability and isolated these non-axisymmetric, oscillatory modes. They showed that for a stationary outer cylinder the most unstable mode is nonaxisymmetric with wave number about five.

We have already commented that fully developed Taylor-Dean flow in the annulus is not easy to construct physically due the difficulty in maintaining azimuthal symmetry. However, the two distinct driving mechanisms of the flow are found together in many practical applications; e.g. an electrogalvanizing line in the steelmaking industry or rotating drum filters in paper- and board-making processes (see [5]). The Taylor-Dean configuration is usually emulated in the laboratory using a partially-filled annulus to produce a combination of Couette and curved Poiseuille flow. There has been considerable interest in the diverse patterns that can be formed from this apparatus, including the coexistence of different states, and Laure \& Mutabazi [6] provide a brief background to this work. Laure \& Mutabazi present a numerical study of the linear and weakly non-linear evolution of the main flow at the onset of instability. Using a partially filled annulus means that symmetry is broken and wavenumbers are no longer necessarily integer. Laure \& Mutabazi [6] demonstrate how this can affect the linear stability analysis and predict regimes of oscillatory and stationary rolls followed by bifurcations via supercritical rather than subcritical transitions.

Kachoyan [4] considered the eigenvalue problem for general values of $\beta$ with particular interest in $\beta>1$ when the pressure gradient acts against rotation. He determined neutral curves associated with both the Taylor-like and Dean-like instabilities (given by the variation of the respective critical Taylor numbers $\widehat{T}_{T}$ and $\widehat{T}_{D}$ ) for several values of $\beta$. Kachoyan observed that, for $\beta=\beta_{0} \simeq 1.275$, the leading order critical Taylor numbers coincide (and the neutral curves meet) at very large wavenumbers. As $\beta$ is increased beyond $\beta_{0}$ the neutral curves associated with the two types of instability appear to cross at finite values of $k$, say $k_{c}$ : for $k<k_{c}$ the Dean instability sets in first in the sense that $\widehat{T}_{D}<\widehat{T}_{T}$; for $k>k_{c}$ the Taylor instability occurs first $\left(\widehat{T}_{D}>\widehat{T}_{T}\right)$. Upon closer examination of the apparent crossing points of the neutral curves Kachoyan concluded that they did not intersect but rather there was a "kink" in each neutral curve.

Here we shall be concerned with large wavenumber perturbations in small-gap Taylor-Dean flow. The aim is to construct a consistent solution to the linear perturbation equations across the whole annular domain for a single Taylor number, corresponding to the concurrent onset of Taylor and Dean instabilities. The objective of this is to explore further the kinking behaviour observed by Kachoyan 
and to place its existence on a firm rational footing. The two possible modes of instability are not treated in isolation but are shown to interact in a subtle way, linked at exponentially small orders by a WKB analysis. There are five crucial regions: a boundary layer at the outer cylinder wall, three internal layers one of which contains the Dean instability and a layer next to the inner (rotating) cylinder wall. We show how it is possible to generate asymptotic series for the Taylor number and wavelength at the concurrent onset of the two types of instability.

In the next section we formulate the problem and identify the different flow regions. In $\S 3$ each zone is considered in turn and asymptotic expansions for the fluid velocity found. Solutions are matched across the critical layers and to the standard boundary conditions at the cylinder walls. In the main body of the fluid the solution is given by a WKB form which links the two instabilities at exponentially small orders and the inclusion of internal critical points results in the appearance of oscillatory solutions. The final section interprets our analysis in the context of the previous study of neutral curve behaviour by Kachoyan.

\section{Notation and fundamental equations}

\subsection{Base flow and linear perturbations}

We consider an incompressible fluid occupying the region between two coaxial cylinders of radii $R_{1}$ and $R_{2}\left(R_{2}=R_{1}+d>R_{1}\right)$. The outer cylinder is at rest while the inner rotates about its axis with angular velocity $\Omega$. In addition we assume that a constant, azimuthal pressure gradient is applied. For a small gap $(d \ll 1)$ there exists a solution to the Navier-Stokes equations given by

$$
p=-\rho \kappa \theta+\rho \int \widehat{u}_{\theta}^{2} r^{-1} \mathrm{~d} r, \quad \widehat{\mathbf{u}}=\widehat{u}_{\theta} \mathbf{e}_{\theta}=\left\{\mathcal{A} r^{-1}+\mathcal{B} r-(4 \nu)^{-1} \kappa r\left(\ln r^{2}-1\right)\right\} \mathbf{e}_{\theta} .
$$

Here $p, \rho, \widehat{\mathbf{u}}$ are the pressure, density and velocity fields respectively, $(r, \theta)$ the usual polar co-ordinates, $\nu$ the kinematic viscosity, $\kappa$ a measure of the pressure gradient and $\mathcal{A}$ and $\mathcal{B}$ are known constants. We non-dimensionalise lengths with respect to $d$, radial and axial velocities by $\nu / 2 d$, time by $d^{2} / \nu$, and $\widehat{u}_{\theta}$ by $V_{T}+V_{D}$ (where $V_{T}=\Omega R_{1} / 2$ and $V_{D}=\kappa d^{2} / 12 \nu$ are typical velocities associated with Taylor and Dean effects). The dimensionless base flow is given by $\mathbf{u}=V \mathbf{e}_{\theta}$ where

$$
V=2(1-\beta)(1-x)+6 \beta x(1-x),
$$

$\beta=V_{D} /\left(V_{T}+V_{D}\right)$ and $x=\left(r-R_{1}\right) / d$ is a new, scaled radial variable. The linearised governing equations for small axisymmetric perturbations, $(u(x), \mathrm{v}(x), w(x)) e^{i k z}$ at neutral stability are

$$
\mathcal{L}^{2} u=\varepsilon^{2} \widehat{T} V \mathrm{v}, \quad \mathcal{L}_{\mathrm{v}}=\frac{1}{2} \varepsilon^{2} u V^{\prime},
$$


where $\varepsilon=k^{-1}, \mathcal{L} \equiv \varepsilon^{2}\left(\mathrm{~d}^{2} / \mathrm{d} x^{2}\right)-1$ and $\widehat{T}=4 d^{3}\left(V_{T}+V_{D}\right)^{2} / v^{2} R_{1}$ is the Taylor number. Here, and henceforth, a dash on a function denotes its derivative with respect to its argument.

We consider high wavenumbers $\varepsilon \ll 1$ and appropriate scalings are $T=\varepsilon^{4} \widehat{T}$, $v=\varepsilon^{-2} \mathrm{v}$. This leads to

$$
\mathcal{L}^{2} u=T V v, \quad \mathcal{L} v=\frac{1}{2} u V^{\prime}
$$

which need to be solved subject to boundary conditions $u=v=u^{\prime}=0$ on each wall $x=0,1$.

\subsection{Flow regions}

The operator $\mathcal{L}$ possesses two implicit scales and suggests that the problem is amenable to a WKB analysis. Critical points arise where $T V V^{\prime} / 2$ takes values 0 or -1 and these require separate consideration. The latter case corresponds to two minima of $V V^{\prime}$ where the Rayleigh criterion is most violated and the onset of instability is expected to occur. The critical points are

$$
\begin{array}{lll}
x_{0}=\frac{1}{6 \beta}\left(4 \beta-1+\frac{1+2 \beta}{\sqrt{3}}\right) & \left(V V^{\prime} \text { minimum }\right), & x_{1}=\frac{4 \beta-1}{6 \beta}\left(V^{\prime}=0\right), \\
x_{2}=\frac{\beta-1}{3 \beta}(V=0), & x_{3}=0\left(V V^{\prime} \text { local minimum }\right) .
\end{array}
$$

The structure at $x_{0}$ corresponds to the onset of a Dean instability and at leading order the Taylor number is given by

$$
T_{D}=-2 /\left.V V^{\prime}\right|_{x_{0}}=\frac{9 \sqrt{3} \beta}{(1+2 \beta)^{2}} .
$$

The location $x_{3}$ corresponds to a Taylor instability at the cylinder wall where

$$
T_{T}=-2 /\left.V V^{\prime}\right|_{x_{3}}=-\frac{1}{2(4 \beta-1)(1-\beta)} .
$$

Therefore there are five clearly defined regions, shown in Figure 1: one near the wall $x=1$ where the boundary conditions must hold; a critical point $x_{0}$ where the Dean instability appears; two internal critical points $x_{1}, x_{2}$ where the WKB solution is no longer appropriate and the wall zone at $x=0$ where the Taylor instability exists.

\subsection{Asymptotic expansions and notation}

We are concerned with the simultaneous onset of Taylor and Dean-like instabilities and their interaction. The question of whether the neutral stability curves 

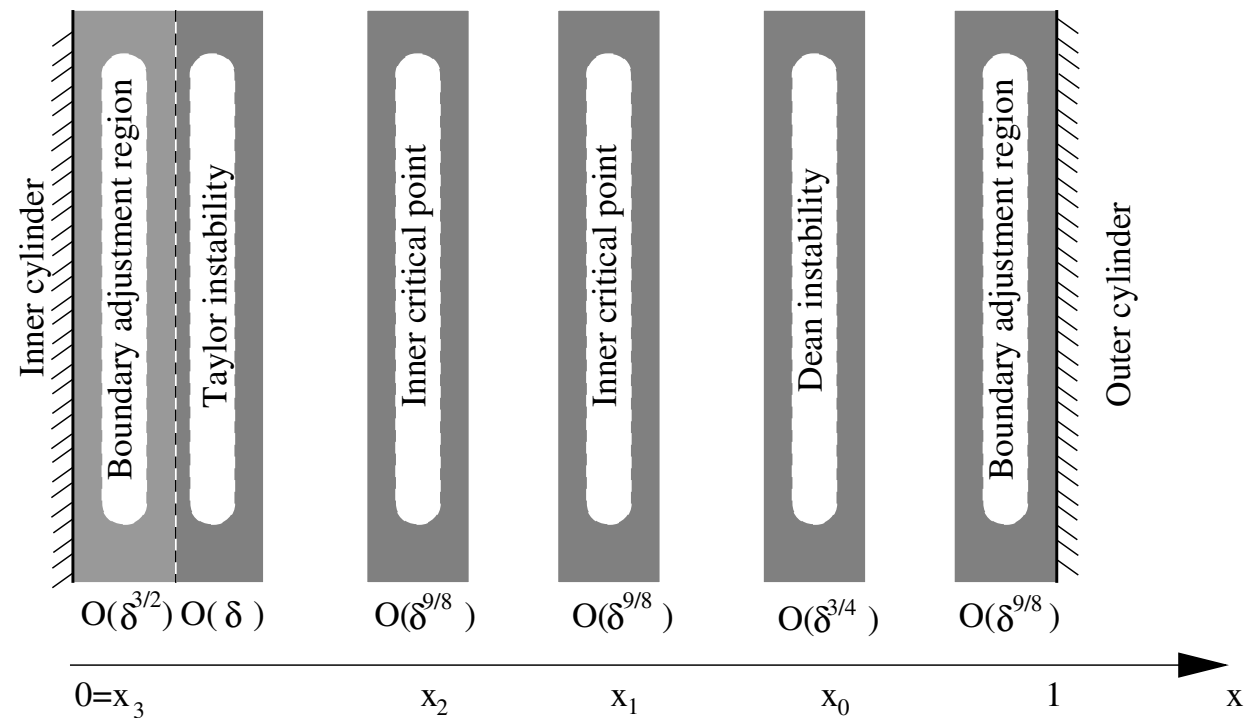

Figure 1. Figure showing asymptotic regions in the Taylor-Dean domain.

corresponding to the onset of each of the two instabilities cross is equivalent to asking whether it is possible to construct a consistent solution across the domain for particular values of the parameters $\beta, \varepsilon$ and Taylor number, $T$.

For the Taylor numbers $T_{D}, T_{T}$ to correspond at leading order we require from equations $(2.6)$ and $(2.7)$ that $\beta=\beta_{0}=(5+3 \sqrt{3}) / 8$, giving $T_{D}=T_{T}=4 / 9$. To match at higher orders we perturb $\beta$ :

$$
\beta=\beta_{0}+\delta, \quad \delta \ll 1 .
$$

Our aim is to construct asymptotic series for the physical quantities of the system in terms of $\delta$ and thereby produce a self-consistent solution in the whole domain. Note that it will not always be necessary to expand functions of $\beta$ in powers of $\delta$ about $\beta_{0}$, but that all series expansions are regular and no ambiguity need arise as long as implicit contributions at higher orders of $\delta$ are acknowledged.

The critical points $x_{0}, x_{3}$ lead to layers of widths $\varepsilon^{1 / 2}$ and $\varepsilon^{2 / 3}$ respectively and the corresponding corrections to the Taylor numbers for the isolated modes of instability are $\mathcal{O}(\varepsilon)$ and $\mathcal{O}\left(\varepsilon^{2 / 3}\right)$. By comparison with the asymptotic forms of (2.6) and (2.7) we need an expansion of $\varepsilon$ given by

$$
\varepsilon=\varepsilon_{0} \delta^{3 / 2}+\varepsilon_{1} \delta^{2}+\ldots
$$

Let us now consider the asymptotic series for the velocities $u, v$ and Taylor number $T$. We shall see that the various critical points are associated with layers of width $\mathcal{O}\left(\delta^{3 / 4}\right), \mathcal{O}(\delta)$ with an embedded layer of $\mathcal{O}\left(\delta^{3 / 2}\right)$, and $\mathcal{O}\left(\delta^{9 / 8}\right)$ and thus 
we expect that velocities $(u, v)$ can be written:

$$
\begin{aligned}
\left(u_{0}, v_{0}\right)+\delta^{1 / 2}\left(u_{1}, v_{1}\right)+\delta^{3 / 4}\left(u_{2}, v_{2}\right) & +\delta\left(u_{3}, v_{3}\right)+\delta^{9 / 8}\left(u_{4}, v_{4}\right) \\
& +\delta^{5 / 4}\left(u_{5}, v_{5}\right)+\delta^{3 / 2}\left(u_{6}, v_{6}\right)+\ldots
\end{aligned}
$$

Other orders of $u$ are not forced by the dynamics of the problem and merely reproduce, by linearity, the main solution. We expand the Taylor number in a similar manner but for later clarity we present two expansions - one appropriate at $x_{0}$, the other at $x_{3}$ :

$$
\begin{aligned}
T & =T_{0}+\delta^{1 / 2} T_{1}+\delta^{3 / 4} T_{2}+\delta T_{3}+\delta^{9 / 8} T_{4}+\delta^{5 / 4} T_{5}+\delta^{3 / 2} T_{6}+\ldots, \\
& =\tau_{0}+\delta^{1 / 2} \tau_{1}+\delta^{3 / 4} \tau_{2}+\delta \tau_{3}+\delta^{9 / 8} \tau_{4}+\delta^{5 / 4} \tau_{5}+\delta^{3 / 2} \tau_{6}+\ldots
\end{aligned}
$$

Note that since the Taylor coefficients (and velocities) will be functions of $\beta, T_{i}$ is not necessarily equal to $\tau_{i}$. Finally we denote the values of $V, V^{\prime}$ at $x_{j}$ by $V_{j}, V_{j}^{\prime}$ respectively.

\section{A linked flow solution throughout the domain}

\subsection{The WKB solution in the main flow}

Consider the flow away from the critical points of $g$ and the boundary walls. There are two scales $x$ and $\varepsilon x$ and we seek a solution of the form

$$
u=\mathrm{u} E, \quad v=\mathrm{v} E \quad \text { where } \quad E \equiv \exp \left\{\varepsilon^{-1} \int^{x} g(s) \mathrm{d} s\right\} .
$$

Neglecting terms of $\mathcal{O}\left(\varepsilon^{3}\right)$, equations (2.4) then yield the system

$$
\begin{gathered}
\left(g^{2}-1\right)^{2} \mathbf{u}+\varepsilon\left[4 \mathbf{u}^{\prime} g\left(g^{2}-1\right)+2 \mathrm{u} g^{\prime}\left(3 g^{2}-1\right)\right] \\
+\varepsilon^{2}\left[2 \mathrm{u}^{\prime \prime}\left(3 g^{2}-1\right)+12 \mathbf{u}^{\prime} g^{\prime} g+\mathbf{u} g^{\prime \prime}(4 g+3)\right]=T v V, \\
\left(g^{2}-1\right) \mathbf{v}+\varepsilon\left(2 \mathbf{v}^{\prime} g+\mathbf{v} g^{\prime}\right)+\varepsilon^{2} \mathbf{v}^{\prime \prime}=\frac{1}{2} \mathbf{u} V^{\prime} .
\end{gathered}
$$

Substituting the asymptotic series for $\mathrm{u}, \mathrm{v}$ and $\varepsilon$ we can equate orders in $\delta$. At $\mathcal{O}(1)$ we find

$$
\left(g^{2}-1\right)^{2} \mathbf{u}_{0}=T_{0} \mathbf{v}_{0} V, \quad\left(g^{2}-1\right) \mathbf{v}_{0}=\frac{1}{2} \mathbf{u}_{0} V^{\prime} \quad \Longrightarrow \quad\left(g^{2}-1\right)^{3}=T_{0} V V^{\prime} / 2 .
$$

Therefore there are six roots which we label

$$
g_{ \pm j}= \pm \sqrt{1+\omega_{j} \operatorname{sgn}\left(\frac{1}{2} T_{0} V V^{\prime}\right)\left|\frac{1}{2} T_{0} V V^{\prime}\right|^{1 / 3}} \text { where } \omega_{j}=e^{2 \pi j \mathrm{i} / 3}, \quad j=0,1,2 .
$$

The solutions $g_{ \pm 0}$ correspond to exponentially growing and decaying solutions whereas the complex solutions are oscillatory with exponentially growing or decaying amplitudes. Figure 2 shows a typical plot of $-\frac{1}{2} T_{0} V V^{\prime}$ and identifies critical points of (3.3) where the WKB form of solution is no longer valid. At $x=x_{1}, x_{2}$, 


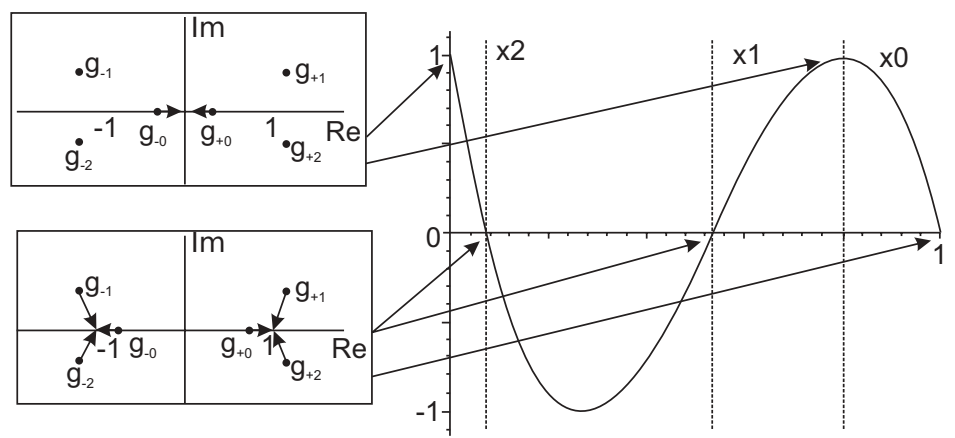

Figure 2. A typical plot of $-T_{0} V V^{\prime} / 2$ showing the critical points of the WKB solution and the behaviour of the roots of $g$ there.

the function $\frac{1}{2} T_{0} V V^{\prime}$ vanishes and each of the three sets of roots $\left\{g_{+j}\right\},\left\{g_{-j}\right\}$ coalesce leaving only two remaining real roots $g= \pm 1$. At $x_{0}$ and $x_{3}, \frac{1}{2} T_{0} V V^{\prime}$ takes the value -1 and the two real roots $g_{ \pm 0}$ coalesce leaving the four remaining complex roots and $g=0$. A sketch of the behaviour of the roots of $g$ in the complex plane is included in figure 2 .

Equating coefficients of $\delta^{1 / 2}, \delta^{3 / 4}, \delta, \delta^{9 / 8}$ and $\delta^{5 / 4}$ we find that, for a consistent solution of (3.2), $T_{1}=T_{2}=T_{3}=T_{4}=T_{5}=0$. This is not surprising - at $x=x_{0}$, $\beta=\beta_{0}$ the correction to the Taylor number is $\mathcal{O}(\varepsilon)$. At $\mathcal{O}\left(\delta^{3 / 2}\right)$ the dynamics are forced by $\mathrm{u}_{0}$ and $\mathrm{v}_{0}$ :

$$
\begin{aligned}
\left(g^{2}-1\right)^{2} \mathbf{u}_{6}+\varepsilon_{0}\left[4 \mathbf{u}_{0}^{\prime} g\left(g^{2}-1\right)+2 \mathbf{u}_{0} g^{\prime}\left(3 g^{2}-1\right)\right] & =T_{0} \mathbf{v}_{6}+T_{6} \mathbf{v}_{0} V, \\
\left(g^{2}-1\right) \mathbf{v}_{6}+\varepsilon_{0}\left(2 \mathbf{v}_{0}^{\prime} g+\mathbf{v}_{0} g^{\prime}\right) & =\frac{1}{2} \mathbf{u}_{6} V^{\prime} .
\end{aligned}
$$

Using (3.3) and (3.5) a first order differential equation for $\mathbf{u}_{0}$ can be determined, namely

$$
\frac{\mathrm{u}_{0}^{\prime}}{\mathrm{u}_{0}}+\frac{g^{\prime}}{2 g}+\frac{V^{\prime \prime}}{3 V^{\prime}}-\frac{T_{6}}{6 \varepsilon_{0} T_{0}} \frac{g^{2}-1}{g}=0 .
$$

Solving (3.6) we find that

$$
\begin{aligned}
u_{0}= & \mathrm{u}_{0} E \propto|g|^{-1 / 2}\left|V^{\prime}\right|^{-1 / 3} \exp \left\{\frac{1}{\varepsilon} \int^{x} g(s) \mathrm{d} s+\frac{T_{6}}{6 \varepsilon_{0} T_{0}} \int^{x} \frac{g(s)^{2}-1}{g(s)} \mathrm{d} s\right\}, \\
v_{0}= & \mathrm{v}_{0} E=\frac{\mathrm{u}_{0} V^{\prime} E}{2\left(g^{2}-1\right)} \propto \\
& \frac{|g|^{-1 / 2}\left|V^{\prime}\right|^{2 / 3}}{2\left(g^{2}-1\right)} \exp \left\{\frac{1}{\varepsilon} \int^{x} g(s) \mathrm{d} s+\frac{T_{6}}{6 \varepsilon_{0} T_{0}} \int^{x} \frac{g(s)^{2}-1}{g(s)} \mathrm{d} s\right\} .
\end{aligned}
$$

The six roots of $g$ give the six linearly independent leading order velocities outside the critical layers. We will link these solutions across the critical layers shown in figure 1 and to the boundaries $x=0,1$. By linearity we assume that both the Taylor and Dean disturbances are $\mathcal{O}(1)$ and that they are spatially decaying, interacting at exponentially small orders via the WKB solutions. 


\subsection{The critical layer at $x_{0}$}

Although as $x_{0}$ is approached the complex roots $g_{ \pm j}, j=1,2$ remain distinct and their velocity contribution is unaffected by the layer, the real roots of $g$ coalesce at 0 . Thus we introduce a scaled variable ${ }^{\dagger}$ given by $\xi=\left(x-x_{0}\right) \delta^{-3 / 4}$, rewrite the differential operator as $\mathcal{L} \equiv \varepsilon^{2} \delta^{-3 / 2}\left(\mathrm{~d}^{2} / \mathrm{d} \xi^{2}\right)-1$ and expand the base velocity about $x_{0}$

$$
V=V_{0}+\delta^{3 / 4} \xi V_{0}^{\prime}+\frac{1}{2} \delta^{3 / 2} \xi^{2} V^{\prime \prime}, \quad V^{\prime}=V_{0}^{\prime}+\delta^{3 / 4} \xi V^{\prime \prime} \quad \text { where } \quad V^{\prime \prime}=-12 \beta .
$$

Substituting expansions for $\varepsilon$ and $(u, v)$ we can equate orders of $\delta$ within $(2.4)$. At $\mathcal{O}(1)$ we have

$$
u_{0}=T_{0} v_{0} V_{0}, \quad-v_{0}=\frac{1}{2} u_{0} V_{0}^{\prime} \quad \Longrightarrow \quad T_{0}=-2 / V_{0} V_{0}^{\prime} .
$$

Thus $T_{0}$ is determined for the neutral stability of Dean disturbances. At the next few orders equations (2.4) reduce to

$$
\begin{gathered}
v_{0}=-\frac{1}{2} u_{0} V_{0}^{\prime}, \quad v_{1}=-\frac{1}{2} u_{1} V_{0}^{\prime}, \quad v_{2}=-\frac{1}{2}\left(u_{2} V_{0}^{\prime}+u_{0} \xi V^{\prime \prime}\right), \\
v_{3}=-\frac{1}{2} u_{3} V_{0}^{\prime}, \quad v_{4}=-\frac{1}{2} u_{4} V_{0}^{\prime}, \quad v_{5}=-\frac{1}{2}\left(u_{5} V_{0}^{\prime}+u_{1} \xi V^{\prime \prime}\right),
\end{gathered}
$$

on noting that $V_{0} V^{\prime \prime}+V_{0}^{\prime} V_{0}^{\prime}=0$. At $\mathcal{O}\left(\delta^{3 / 2}\right)$ we have a linear equation governing $u_{0}$ :

$$
\frac{d^{2} u_{0}}{d \xi^{2}}+\frac{u_{0}}{3 \varepsilon_{0}^{2}}\left(\frac{T_{6}}{T_{0}}+\frac{3 V^{\prime \prime}}{2 V_{0}} \xi^{2}\right)=0 .
$$

Equation (3.11) reduces to the parabolic cylinder equation

$\frac{\mathrm{d}^{2} u_{0}}{\mathrm{~d} X^{2}}-u_{0}\left(a+\frac{1}{4} X^{2}\right)=0 \quad$ where $X=\left(\frac{-2 V^{\prime \prime}}{\varepsilon_{0}^{2} V_{0}}\right)^{1 / 4} \xi, \quad a=-\frac{T_{6}}{3 \varepsilon_{0} T_{0}}\left(\frac{V_{0}}{-2 V^{\prime \prime}}\right)^{1 / 2}$,

which possesses two linearly independent solutions $U(a, X), V(a, X)$. The asymptotic forms of these solutions for $X \gg 1$ are given by Abramowitz \& Stegun [7] as

$$
\begin{aligned}
U(a, X) & \sim X^{-a-\frac{1}{2}} e^{-X^{2} / 4}, \\
U(a,-X) & =\frac{\pi}{\Gamma\left(\frac{1}{2}+a\right)} V(a, X)-\sin \pi a U(a, X), \\
V(a, X) & \sim \sqrt{\frac{2}{\pi}} X^{a-\frac{1}{2}} e^{X^{2} / 4}, \\
V(a,-X) & =\frac{\Gamma\left(\frac{1}{2}+a\right) \cos ^{2} \pi a}{\pi} U(a, X)+\sin \pi a V(a, x) .
\end{aligned}
$$

We are investigating the (concurrent) onset of instability and assume that any deviation from the isolated $\mathcal{O}(1)$ Taylor and Dean modes of instability is tiny.

\footnotetext{
$\dagger$ The same variable will be used in all critical layers with its definition implicit from the context.
} 
Thus we reject any contribution to $u_{0}$ from $V(a, X)$ as it displays exponential growth as $X \rightarrow \infty$. By linearity we take $u_{0}=U(a, X)$ so

$$
\begin{aligned}
& u_{0} \sim X^{-a-\frac{1}{2}} e^{-X^{2} / 4}, \quad \text { as } X \rightarrow \infty, \\
& u_{0} \sim \frac{\sqrt{2 \pi}}{\Gamma\left(\frac{1}{2}+a\right)}(-X)^{a-\frac{1}{2}} e^{X^{2} / 4}-\sin \pi a(-X)^{-a-\frac{1}{2}} e^{-X^{2} / 4}, \quad \text { as } X \rightarrow-\infty .
\end{aligned}
$$

Although the solution seems to possess a term with exponential growth as $X \rightarrow$ $-\infty$, at $a=-1 / 2$ its coefficient vanishes, corresponding to an isolated Dean mode of instability. We require that $a$ be exponentially close to $-1 / 2$ and that any growth will become $\mathcal{O}(1)$ at $x=x_{3}$, corresponding to the interaction between the Taylor and Dean modes. This condition will relate $T_{6}$ and $\varepsilon_{0}$.

We match this layer onto the main flow for $x_{1}<x<x_{0}$ using the method of intermediate scales. Introducing a lower limit, $l_{0}=x_{0}-\delta^{3 / 4}$ to accommodate the simple pole of the integrand, we find that the radial velocity for $x_{1}<x<x_{0}$ is given by

$$
\begin{aligned}
u_{0} & =\mathcal{C}_{+}\left|g_{+0}\right|^{-1 / 2}\left|V^{\prime}\right|^{-1 / 3} \exp \left\{\frac{1}{\varepsilon} \int_{x_{0}}^{x} g_{+0}(s) \mathrm{d} s+\frac{T_{6}}{6 \varepsilon_{0} T_{0}} \int_{l_{0}}^{x} \frac{g_{+0}(s)^{2}-1}{g_{+0}(s)} \mathrm{d} s\right\} \\
& +\mathcal{C}_{-}\left|g_{-0}\right|^{-1 / 2}\left|V^{\prime}\right|^{-1 / 3} \exp \left\{\frac{1}{\varepsilon} \int_{x_{0}}^{x} g_{-0}(s) \mathrm{d} s+\frac{T_{6}}{6 \varepsilon_{0} T_{0}} \int_{l_{0}}^{x} \frac{g_{-0}(s)^{2}-1}{g_{-0}(s)} \mathrm{d} s\right\}
\end{aligned}
$$

where $\alpha=-2 V_{0} / V^{\prime \prime}$ with

$$
\begin{aligned}
& \mathcal{C}_{+}=-\sin \pi a \alpha^{-1 / 4}\left(-V_{0}^{\prime}\right)^{1 / 3} \delta^{3 / 8}\left(\frac{4}{\varepsilon_{0}^{2} \alpha}\right)^{-\frac{1}{4}\left(a-\frac{1}{2}\right)}, \\
& \mathcal{C}_{-}=\frac{\sqrt{2 \pi}}{\Gamma\left(\frac{1}{2}+a\right)} \alpha^{-1 / 4}\left(-V_{0}^{\prime}\right)^{1 / 3} \delta^{3 / 8}\left(\frac{4}{\varepsilon_{0}^{2} \alpha}\right)^{\frac{1}{4}\left(a-\frac{1}{2}\right)} .
\end{aligned}
$$

We note that the factor $\delta^{3 / 8}$ implies that the flow becomes more intense in the layer, as might be expected. There will be contributions to the velocity from the solutions associated with the complex roots of $g$ but, as we shall see, in this region they are negligible.

\subsection{The critical layer about $\mathrm{x}_{1}$}

In the main flow the leading order velocities $u_{0}, v_{0}$ are proportional to $\left|V^{\prime}\right|^{-1 / 3}$ and $\left|V^{\prime}\right|^{2 / 3}\left(g^{2}-1\right)^{-1}$ respectively. As we approach $x=x_{1}$ we find that $V^{\prime} \rightarrow 0$, $g \rightarrow \pm 1$ and $\left|V^{\prime}\right|^{2 / 3}\left(g^{2}-1\right)^{-1} \rightarrow 0$. It is clear therefore that, near this critical point, the assumption that $u_{0}, v_{0}$ are of the same order is no longer valid. By careful 
consideration of the appropriate asymptotic scales and the governing equations it can be seen that, near $x_{1}, u \sim \mathcal{O}\left(\delta^{-3 / 8}\right), v \sim \mathcal{O}\left(\delta^{3 / 8}\right)$ in a layer of width $\mathcal{O}\left(\delta^{9 / 8}\right)$.

We introduce the scaled variable $\xi=\left(x-x_{1}\right) \delta^{-9 / 8}$ and look for solutions of the form

$$
u=\delta^{-3 / 8} \widetilde{u}(\xi) \exp ( \pm x / \varepsilon), \quad v=\delta^{3 / 8} \widetilde{v}(\xi) \exp ( \pm x / \varepsilon) .
$$

The velocity $V$ and operator $\mathcal{L}$ can be expressed in terms of $\xi$, in the same manner as $\S 3.2$, so that the governing equations about $x_{1}$ become

$$
\begin{aligned}
& 4 \varepsilon^{2} \delta^{-9 / 4} \widetilde{u}^{\prime \prime}+\mathcal{O}\left(\varepsilon^{3}\right)=\delta^{3 / 4} T \widetilde{v}\left(V_{1}+\frac{1}{2} \delta^{9 / 4} \xi^{2} V^{\prime \prime}\right) \\
& \pm 2 \varepsilon \delta^{-9 / 8} \widetilde{v}^{\prime}+\mathcal{O}\left(\varepsilon^{2}\right)=\frac{1}{2} \delta^{3 / 8} \xi V^{\prime \prime} \widetilde{u}
\end{aligned}
$$

Expanding $\widetilde{u}(\xi), \widetilde{v}(\xi)$ according to (2.10) and equating at lowest orders of $\delta$, we find that

$$
\widetilde{u}_{0}^{\prime \prime \prime}= \pm \frac{T_{0} V_{1} V^{\prime \prime}}{16 \varepsilon_{0}^{3}} \xi \widetilde{u}_{0}, \quad \widetilde{v}_{0}=\frac{4 \varepsilon_{0}^{2}}{T_{0} V_{1}} \widetilde{u}_{0}^{\prime \prime} .
$$

A change of variables simplifies the system within the layer to two third order differential equations for the radial velocities corresponding to the two sets of roots, $\left\{g_{+j}\right\}$ and $\left\{g_{-j}\right\}$ outside the layer:

$\frac{\mathrm{d}^{3} \widetilde{u}_{0}}{\mathrm{~d} X_{ \pm}^{3}}=-X_{ \pm} \widetilde{u}_{0}, \quad$ where $\quad X_{+}=\left(2 \varepsilon_{0}\right)^{-3 / 4} \gamma^{1 / 4} \xi=e^{-\pi \mathrm{i} / 4} X_{-}, \quad \gamma=-\frac{1}{2} T_{0} V_{1} V^{\prime \prime}$

In the limit $\left|X_{ \pm}\right| \gg 1$ there are three linearly independent solutions with asymptotic forms

$$
f_{ \pm j}=X_{ \pm}^{-1 / 3} \exp \left\{-\frac{3}{4} \omega_{j} X_{ \pm}^{4 / 3}\right\}, \quad j=0,1,2
$$

and our solution will match a linear combination of the $f_{ \pm j}$. But, to obtain a consistent solution to (3.20) throughout the complex plane for $\left|X_{ \pm}\right| \gg 1$, we require that, as the argument of $X_{ \pm}$varies (and we cross the layer), the coefficients of the $f_{ \pm j}$ change as we cross Stokes lines. The changes are given by known Stokes multipliers. The asymptotic behaviour of equation (3.20) has been analysed by Heading [8] and as $\arg X_{+}$goes from $0_{+}$to $\pi_{-}$the linear combination $c_{0} f_{+0}+$ $c_{1} f_{+1}+c_{2} f_{+2}$ becomes $c_{0} f_{+0}+c_{1} f_{+1}+\left(e^{2 \pi \mathrm{i} / 3} c_{0}+e^{\pi \mathrm{i} / 3} c_{1}+c_{2}\right) f_{+2}$. Similarly, as $\arg X_{-}$goes from $\pi / 4$ to $5 \pi / 4, c_{0} f_{-0}+c_{1} f_{-1}+c_{2} f_{-2}$ becomes $c_{0} f_{-0}+e^{2 \pi \mathrm{i} / 3} c_{2} f_{-1}+$ $\left(e^{2 \pi \mathrm{i} / 3} c_{0}+e^{\pi \mathrm{i} / 3} c_{1}+c_{2}\right) f_{-2}$. We now trace the evolution of our solution (3.15) across $x=x_{1}$. Note that we only consider the evolution of two of the six exponential solutions through the layer. We discuss the contribution of the other four terms later and it is clear that the linearity of the problem allows such an independent treatment.

Using intermediate scales, we find that to the right of the layer the matched solution can be written

$$
\widetilde{\mathcal{C}}_{+}\left(2 \varepsilon_{0}\right)^{-1 / 4} \gamma^{1 / 12} \delta^{-3 / 8} f_{+0} e^{x / \varepsilon}+\widetilde{\mathcal{C}}_{-}\left(2 \varepsilon_{0}\right)^{-1 / 4} e^{\pi \mathrm{i} / 12} \gamma^{1 / 12} \delta^{-3 / 8} f_{-1} e^{-x / \varepsilon}
$$


where

$$
\widetilde{\mathcal{C}}_{ \pm}=\mathcal{C}_{ \pm}\left(-V^{\prime \prime}\right)^{-1 / 3} \exp \left\{\frac{1}{\varepsilon} \int_{x_{0}}^{x_{1}} g_{ \pm 0} \mathrm{~d} s \mp \frac{x_{1}}{\varepsilon}+\frac{T_{6}}{6 \varepsilon_{0} T_{0}} \int_{l_{0}}^{x_{1}} \frac{g_{ \pm 0}^{2}-1}{g_{ \pm 0}} \mathrm{~d} s\right\} .
$$

Thus, using the Stokes multipliers given above, the solution as we move to the left of $x_{1}$ is

$$
\begin{aligned}
\widetilde{\mathcal{C}}_{+}\left(2 \varepsilon_{0}\right)^{-1 / 4} \gamma^{1 / 12} \delta^{-3 / 8}\left(f_{+0}+\right. & \left.e^{2 \pi \mathrm{i} / 3} f_{+2}\right) e^{x / \varepsilon} \\
& +\widetilde{\mathcal{C}}_{-}\left(2 \varepsilon_{0}\right)^{-1 / 4} e^{5 \pi \mathrm{i} / 12} \gamma^{1 / 12} \delta^{-3 / 8} f_{-2} e^{-x / \varepsilon} .
\end{aligned}
$$

In other words, the dominant solution associated with $\left(g_{-0}+1\right)-$ an increasing function about $x_{1}$ - remains dominant and the sub-dominant solution relating to $\left(g_{+0}-1\right)$ - a decreasing function about $x_{1}$ - becomes a pair of oscillating solutions. Thus we can match onto a WKB solution in the region $x_{2}<x<x_{1}$ :

$$
\begin{aligned}
u_{0}= & \mathcal{D}_{+}\left|g_{+1}\right|^{-1 / 2}\left|V^{\prime}\right|^{-1 / 3} \exp \left\{\frac{1}{\varepsilon} \int_{x_{1}}^{x} g_{+1}(s) \mathrm{d} s+\frac{T_{6}}{6 \varepsilon_{0} T_{0}} \int_{x_{1}}^{x} \frac{g_{+1}(s)^{2}-1}{g_{+1}(s)} \mathrm{d} s\right\}+\text { c.c. } \\
& +\mathcal{D}_{-}\left|g_{-0}\right|^{-1 / 2}\left|V^{\prime}\right|^{-1 / 3} \exp \left\{\frac{1}{\varepsilon} \int_{x_{1}}^{x} g_{-0}(s) \mathrm{d} s+\frac{T_{6}}{6 \varepsilon_{0} T_{0}} \int_{x_{1}}^{x} \frac{g_{-0}(s)^{2}-1}{g_{-0}(s)} \mathrm{d} s\right\},
\end{aligned}
$$

where

$$
\begin{aligned}
& \mathcal{D}_{+}=\mathcal{C}_{+} e^{\pi \mathrm{i} / 3} \exp \left\{\frac{1}{\varepsilon} \int_{x_{0}}^{x_{1}} g_{+0} \mathrm{~d} s+\frac{T_{6}}{6 \varepsilon_{0} T_{0}} \int_{l_{0}}^{x_{1}} \frac{g_{+0}^{2}-1}{g_{+0}} \mathrm{~d} s\right\}, \\
& \mathcal{D}_{-}=\mathcal{C}_{-} \exp \left\{\frac{1}{\varepsilon} \int_{x_{0}}^{x_{1}} g_{-0} \mathrm{~d} s+\frac{T_{6}}{6 \varepsilon_{0} T_{0}} \int_{l_{0}}^{x_{1}} \frac{g_{-0}^{2}-1}{g_{-0}} \mathrm{~d} s\right\} .
\end{aligned}
$$

\subsection{The critical layer about $\mathrm{x}_{2}$}

The layer about $x_{2}$ is analogous to that at $x_{1}$, except that now it is $V$ that vanishes rather than $V^{\prime}$. The appropriate scaling is $u_{0} \sim \mathcal{O}(1)$ and, since $g \rightarrow$ $\pm 1, v_{0} \sim \mathcal{O}\left(\delta^{-3 / 8}\right)$ within a layer of $\mathcal{O}\left(\delta^{9 / 8}\right)$. We introduce the new variable $\xi=\left(x-x_{2}\right) \delta^{-9 / 8}$ and write $u=\widetilde{u}(\xi) e^{ \pm x / \varepsilon}, v=\delta^{-3 / 8} \widetilde{v}(\xi) e^{ \pm x / \varepsilon}$ whereupon the governing equations can be written

$$
\begin{aligned}
& 4 \varepsilon^{2} \delta^{-9 / 4} \widetilde{u}^{\prime \prime}+\mathcal{O}\left(\varepsilon^{3}\right)=T \delta^{-3 / 8} \widetilde{v}\left(\delta^{9 / 8} \xi V_{2}^{\prime}+\frac{1}{2} \delta^{9 / 4} \xi^{2} V^{\prime \prime}\right) \\
& \pm 2 \varepsilon \delta^{-9 / 8} \widetilde{v}^{\prime}+\mathcal{O}\left(\varepsilon^{2}\right)=\frac{1}{2} \delta^{3 / 8} \widetilde{u}\left(V_{2}^{\prime}+\delta^{9 / 8} \xi V^{\prime \prime}\right) .
\end{aligned}
$$

We find that the azimuthal velocity satisfies the same differential equation as we found above for the radial perturbation velocity,

$\frac{\mathrm{d}^{3} \widetilde{v}_{0}}{\mathrm{~d} X_{ \pm}^{3}}=-X_{ \pm} \widetilde{v}_{0}, \quad$ where $\quad X_{-}=\left(2 \varepsilon_{0}\right)^{-3 / 4} \lambda^{1 / 4} \xi=e^{-\pi \mathrm{i} / 4} X_{+}, \quad \lambda=-\frac{1}{2} T_{0}\left(V_{2}^{\prime}\right)^{2}$, 
and $\widetilde{u}_{0}=4 \varepsilon_{0} \widetilde{v}_{0}^{\prime} / V_{2}^{\prime}$. Therefore the asymptotic solutions are given by $\left\{f_{ \pm j}\right\}$, see (3.21), and their variation through the complex plane is determined by the same Stokes multipliers. We find, using $(3.7 \mathrm{~b})$, that as we match to the right we have an incoming WKB solution of the form

$$
\begin{aligned}
\widetilde{v}_{0}=\left(\widetilde{\mathcal{D}}_{+} f_{+2}+\widetilde{\mathcal{D}}_{+}^{*} f_{+0}\right)\left(2 \varepsilon_{0}\right)^{-1 / 4} e^{\pi \mathrm{i} / 12} \lambda^{1 / 12} \delta^{-3 / 8} e^{-x / \varepsilon} & \\
& +\widetilde{\mathcal{D}}_{-}\left(2 \varepsilon_{0}\right)^{-1 / 4} \lambda^{1 / 12} \delta^{-3 / 8} f_{-0} e^{-x / \varepsilon}
\end{aligned}
$$

where

$$
\begin{aligned}
& \widetilde{\mathcal{D}}_{+}=\mathcal{D}_{+} \omega_{2}\left(V_{2}^{\prime}\right)^{-4 / 3} T_{0}^{-1} \lambda^{2 / 3} \exp \left\{\frac{1}{\varepsilon} \int_{x_{1}}^{x_{2}} g_{+1} \mathrm{~d} s-\frac{x_{2}}{\varepsilon}+\frac{T_{6}}{6 \varepsilon_{0} T_{0}} \int_{x_{1}}^{x_{2}} \frac{g_{+1}^{2}-1}{g_{+1}} \mathrm{~d} s\right\} \\
& \widetilde{\mathcal{D}}_{-}=\mathcal{D}_{-}\left(V_{2}^{\prime}\right)^{-4 / 3} T_{0}^{-1} \lambda^{2 / 3} \exp \left\{\frac{1}{\varepsilon} \int_{x_{1}}^{x_{2}} g_{-0} \mathrm{~d} s+\frac{x_{2}}{\varepsilon}+\frac{T_{6}}{6 \varepsilon_{0} T_{0}} \int_{x_{1}}^{x_{2}} \frac{g_{-0}^{2}-1}{g_{-0}} \mathrm{~d} s\right\}
\end{aligned}
$$

and $*$ indicates complex conjugate. As $\xi$ goes from $+\infty$ to $-\infty$, so $\arg X_{+}$goes from $\pi / 4$ to $5 \pi / 4$ and $\arg X_{-}$from 0 to $\pi$. Near $x_{2}, g_{-0}$ is decreasing about -1 and thus $f_{-0}$ is the sub-dominant solution. Therefore when we match to the left this solution maps onto two oscillating solutions whereas the oscillating solutions associated with $g_{+1,2}$ are equally dominant and merge into a single solution. Matching the solution for $\widetilde{v}_{0}$ in the layer about $x_{2}$ to the left, it can be shown that in the region $x_{3}<x<x_{2}$, the radial velocity satisfies

$$
\begin{aligned}
& u_{0}=\mathcal{E}_{+}\left|g_{+0}\right|^{-1 / 2}\left|V^{\prime}\right|^{-1 / 3} \exp \left\{\frac{1}{\varepsilon} \int_{x_{2}}^{x} g_{+0}(s) \mathrm{d} s+\frac{T_{6}}{6 \varepsilon_{0} T_{0}} \int_{x_{2}}^{x} \frac{g_{+0}(s)^{2}-1}{g_{+0}(s)} \mathrm{d} s\right\} \\
+ & \mathcal{E}_{-}\left|g_{-1}\right|^{-1 / 2}\left|V^{\prime}\right|^{-1 / 3} \exp \left\{\frac{1}{\varepsilon} \int_{x_{2}}^{x} g_{-1}(s) \mathrm{d} s+\frac{T_{6}}{6 \varepsilon_{0} T_{0}} \int_{x_{2}}^{x} \frac{g_{-1}(s)^{2}-1}{g_{-1}(s)} \mathrm{d} s\right\}+\text { c.c. },
\end{aligned}
$$

where

$$
\begin{aligned}
& \mathcal{E}_{+}=\mathcal{D}_{+} \exp \left\{\frac{1}{\varepsilon} \int_{x_{1}}^{x_{2}} g_{+1} \mathrm{~d} s+\frac{T_{6}}{6 \varepsilon_{0} T_{0}} \int_{x_{1}}^{x_{2}} \frac{g_{+1}^{2}-1}{g_{+1}} \mathrm{~d} s\right\}+\text { c.c. } \\
& \mathcal{E}_{-}=\mathcal{D}_{-} \exp \left\{\frac{1}{\varepsilon} \int_{x_{1}}^{x_{2}} g_{-0} \mathrm{~d} s+\frac{T_{6}}{6 \varepsilon_{0} T_{0}} \int_{x_{1}}^{x_{2}} \frac{g_{-0}^{2}-1}{g_{-0}} \mathrm{~d} s\right\} .
\end{aligned}
$$

\subsection{The layer at $\mathrm{x}_{3}=0$}

We now study the final critical point $x_{3}=0$, a local minimum of $\frac{1}{2} T_{0} V V^{\prime}$. It is not a stationary point of the function and hence the layer is of width $\mathcal{O}(\delta)$. The governing system becomes

$$
\mathcal{L}^{2} u=T v\left(V_{3}+\delta \xi V_{3}^{\prime}+\frac{1}{2} \delta^{2} \xi^{2} V^{\prime \prime}\right), \quad \mathcal{L} v=\frac{1}{2} u\left(V_{3}^{\prime}+\delta \xi V^{\prime \prime}\right)
$$


where $\xi=x \delta^{-1}$ and the operator $\mathcal{L}=\varepsilon^{2} \delta^{-2}\left(\mathrm{~d}^{2} / \mathrm{d} \xi^{2}\right)-1$. We can equate coefficients in $\delta$ but, as will become apparent, it is necessary to use the asymptotic expansion of $T$ in terms of $\tau_{j}$. We find

$$
u_{0}=\tau_{0} v_{0} V_{3}, \quad-v_{0}=\frac{1}{2} u_{0} V_{3}^{\prime} \quad \Longrightarrow \quad \tau_{0}=-1 / 2 V_{3} V_{3}^{\prime} ;
$$

at higher orders the equations reduce to $\tau_{1}=\tau_{2}=0$. The problem is forced at $\mathcal{O}(\delta)$ where

$$
u_{3}-2 \varepsilon_{0}^{2} u_{0}^{\prime \prime}=\tau_{0} V_{3} v_{3}+\tau_{0} \xi V_{3}^{\prime} v_{0}+\tau_{3} V_{3} v_{0}, \quad \varepsilon_{0}^{2} v_{0}^{\prime \prime}-v_{3}=\frac{1}{2} \xi V^{\prime \prime} u_{0}+\frac{1}{2} V_{3}^{\prime} u_{3} .
$$

Note that $T_{0}, \tau_{0}$ only agree up to $\mathcal{O}(\delta)$ and hence $\tau_{3} \neq 0$. Using (3.34), system (3.35) reduces to

$$
\frac{\mathrm{d}^{2} u_{0}}{\mathrm{~d} X^{2}}=X u_{0}
$$

where

$$
X=\lambda_{0}^{1 / 3} \xi-\lambda_{1} \lambda_{0}^{-2 / 3}, \quad \lambda_{0}=-\frac{1}{3 \varepsilon_{0}^{2}}\left(\frac{V^{\prime \prime}}{V_{3}^{\prime}}+\frac{V_{3}^{\prime}}{V_{3}}\right)>0, \quad \lambda_{1}=\frac{\tau_{3}}{3 \tau_{0} \varepsilon_{0}^{2}} .
$$

The Airy functions $\operatorname{Ai}(X)$ and $\operatorname{Bi}(X)$ solve (3.36) and their asymptotic behaviours for $X \gg 1$ are

$$
\operatorname{Ai}(X) \sim 2^{-1} \pi^{-1 / 2} X^{-1 / 4} \exp \left(-\frac{2}{3} X^{3 / 2}\right), \quad \operatorname{Bi}(X) \sim \pi^{-1 / 2} X^{-1 / 4} \exp \left(\frac{2}{3} X^{3 / 2}\right) .
$$

It is clear that $\mathrm{Bi}$ is exponentially increasing and cannot contribute at $\mathcal{O}(1)$ as this would not correspond to an isolated mode of instability. The coefficient of Bi must be exponentially small indicating a slight detuning of the isolated mode and linking with the incoming WKB mode corresponding to $g_{+0}$. Thus we find the leading order contribution to $\mathrm{Bi}$ is

$$
\frac{\mathcal{E}_{+}}{\sqrt{\pi \varepsilon_{0}}} \delta^{1 / 4} \lambda_{0}^{-1 / 6}\left(V_{3}^{\prime}\right)^{-1 / 3} \exp \left\{\frac{1}{\varepsilon} \int_{x_{2}}^{0} g_{+0} \mathrm{~d} s+\frac{T_{6}}{6 \varepsilon_{0} T_{0}} \int_{x_{2}}^{0} \frac{g_{+0}^{2}-1}{g_{+0}} \mathrm{~d} s\right\} \operatorname{Bi}(X) .
$$

We expect the coefficient of Ai to be $\mathcal{O}(1)$ as we wish the two instabilities, Taylor and Dean, to occur simultaneously and be of similar sizes. It is clear that as $X \rightarrow \infty$ this links to the velocity corresponding with the root $g_{-0}$ in the region $x_{3}<x<x_{2}$ which, as it encounters the critical point $x_{2}$ from the left, will become two oscillatory, complex conjugate solutions corresponding to $g_{-1,2}$. These will continue to decrease exponentially to the wall $x=1$. Thus the coefficient of $\mathrm{Ai}$ is not known and we can only say at this stage that $u_{0}=\mathcal{F} \operatorname{Ai}(X)$ where $\mathcal{F}$ is some $\mathcal{O}(1)$ constant.

We hope to investigate corrections to the Taylor number up to $\mathcal{O}\left(\delta^{3 / 2}\right)$ and continue to equate at higher orders of $\delta$. At $\mathcal{O}\left(\delta^{9 / 8}\right), \mathcal{O}\left(\delta^{5 / 4}\right)$ we find that $\tau_{4}=$ $\tau_{5}=0$. But, at $\mathcal{O}\left(\delta^{3 / 2}\right)$, we find

$$
\begin{aligned}
u_{6}-2 \varepsilon_{0}^{2} u_{1}^{\prime \prime}-4 \varepsilon_{0} \varepsilon_{1} u_{0}^{\prime \prime} & =\tau_{0} v_{1} \xi V_{3}^{\prime}+\tau_{0} v_{6} V_{3}+\tau_{3} v_{1} V_{3}, \\
\varepsilon_{0}^{2} v_{1}^{\prime \prime}+2 \varepsilon_{0} \varepsilon_{1} v_{0}^{\prime \prime}-v_{6} & =\frac{1}{2} V_{3}^{\prime} u_{6}+\frac{1}{2} V^{\prime \prime} \xi u_{1}^{\prime \prime} .
\end{aligned}
$$


Using the same change of variables as (3.36) above, equation (3.39) can be rewritten as

$$
\frac{\mathrm{d}^{2} u_{1}}{\mathrm{~d} X^{2}}-X u_{1}=-\frac{\tau_{6}}{3 \varepsilon_{0}^{2} \lambda_{0}^{2 / 3}} u_{0}+\frac{2 \varepsilon_{1}}{\varepsilon_{0}} \frac{\mathrm{d}^{2} u_{0}}{\mathrm{~d} X^{2}},
$$

which is solved in terms of Airy functions to give

$$
u_{1}=\mathcal{G} \operatorname{Ai}(X)-\left(\frac{\tau_{6}}{3 \varepsilon_{0}^{2} \lambda_{0}^{2 / 3}}+\frac{2 \varepsilon_{1} X}{3 \varepsilon_{0}}\right) \mathcal{F} \operatorname{Ai}^{\prime}(X) .
$$

Before we can write down the final conditions governing the parameters of our linked leading order solutions we must consider the boundary conditions at the two cylinder walls $x=0,1$.

\subsection{Boundary conditions at $x=0$ and $x=1$}

The boundary conditions $u=v=w=0$ on $x=0,1$ are equivalent to demanding that $u=u^{\prime}=\mathcal{L}^{2} u=0$ at these locations. Near $x=1$ the flow has decayed to be exponentially small but even here it is necessary to ensure adherence to the boundary conditions. We have found six linearly independent solutions across the flow domain but it is clear that at any position in the fluid not all these solutions can exist at leading order. The condition that the flow should only be $\mathcal{O}(1)$ at the two points of instability coupled with the boundary conditions allows us to anticipate the dominant flow solutions across the domain and, in particular, at the cylinder boundaries. Figure 3 shows diagrammatically the evolution of the six flow elements. The different styles of line illustrate the linking of solutions and the legend distinguishes the cartoons used to depict each of the six types of solution. An indication of the magnitude of a solution is given by its vertical position.

We begin by considering the wall at $x=1$. Of the six solutions here, the two oscillatory decaying solutions associated with $g_{-1,2}$ link directly to the decay of the Airy solution at $x_{3}$. As such they have decayed throughout the whole fluid domain and at $x=1$ we would expect them to be far smaller than the other flow components. Thus to satisfy the boundary conditions we have four remaining solutions at our disposal: two oscillating solutions associated with $g_{+1,2}$ which decay all the way to the wall $x=0$; one exponentially increasing towards $x_{0}$ associated with the $\mathcal{O}(1)$ Dean instability; and the solution associated with $g_{+0}$ which links with the solution $V(a, X)$ of the parabolic cylinder equation. The coefficient of the solution associated with $g_{-0}$ is predetermined by our choice of coefficient for $U(a, X)$. Hence we require all three of the remaining solutions to be at most the same order as the $g_{-0}$ solution at the boundary $x=1$ in order to satisfy the boundary conditions. Evidently this means that their magnitudes will be exponentially small at $x=1$. Consequently when the solution associated with $g_{+0}$ reaches $x_{0}$ it has further decayed exponentially and the coefficient of $V(a, X)$ will be extremely small. Thus, although $V(a, X)$ does display exponential growth 


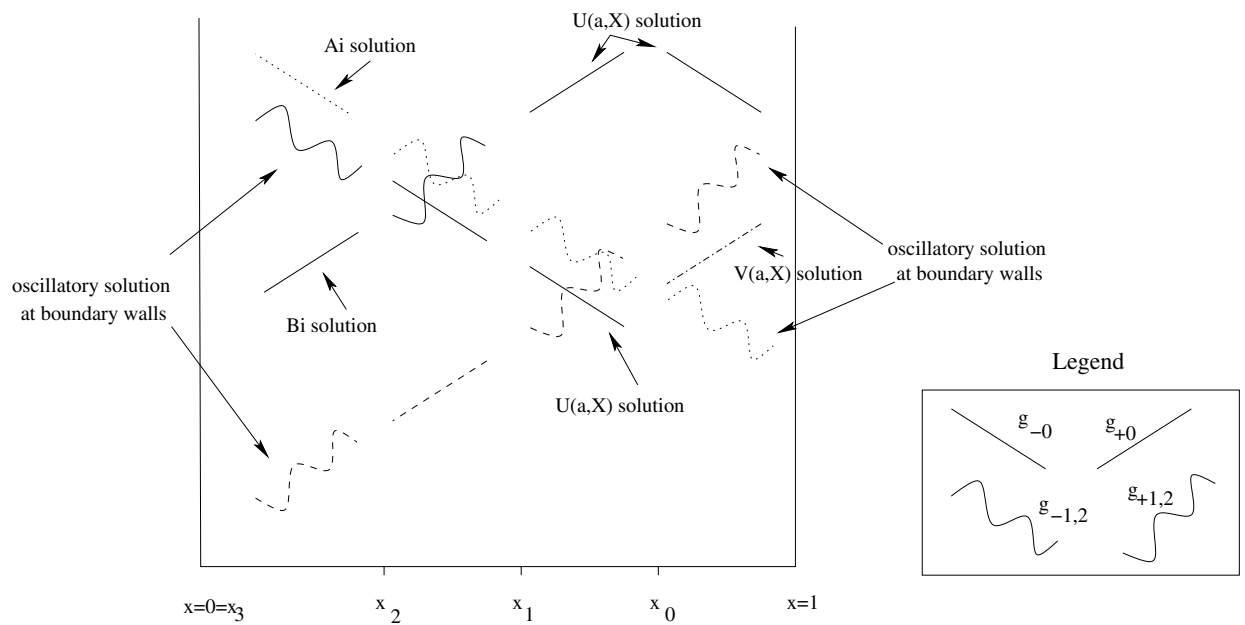

Figure 3. Diagram of the six linearly independent solutions across the flow domain.

in both directions $X \rightarrow \pm \infty$, it can still be subordinate to $U(a, X)$ at $x_{0}$ and will not affect the interaction of our leading order solutions. Hence we do not continue its evolution in figure 3.

Near $x=1$ the system is analogous to the layer at $x_{2}$ with $V \rightarrow 0$ and $g_{ \pm j} \rightarrow$ \pm 1 . Rescaling we find that the two sets of solutions are governed by the third order differential equation studied by Heading [8]. As we approach $x=1$ the solutions can be approximated by generalised hypergeometric functions. The coefficient of the solution associated with $g_{-0}$ is fixed by matching with the Dean instability and the three coefficients corresponding to $g_{+0}, g_{-j}$ can be chosen to satisfy the boundary conditions.

The boundary $x=0$ requires more detailed consideration. Examining figure 3 we see that of the six solutions, the exponentially increasing one associated with $\mathrm{Bi}$ is linked to the Dean instability and hence is exponentially small. The oscillatory growing modes link with the exponentially small oscillating modes at the wall $x=1$ and have further decayed across the domain. The only remaining, possibly $\mathcal{O}(1)$, modes are the oscillatory decaying solutions $g_{-j}$ (also linking to the Dean instability $U(a, X))$ and the decreasing solution associated with Ai. However we have three homogeneous boundary conditions and the three dominant solutions will be unable to satisfy them non-trivially. Hence an inner adjustment layer is introduced to satisfy the boundary conditions.

\subsection{The inner layer at $\mathrm{x}=0$}

The inner layer at $x=0$ is of width $\mathcal{O}\left(\delta^{3 / 2}\right)$. With $\xi \equiv x \delta^{-3 / 2}$, we find that $\mathcal{L} \equiv \varepsilon^{2} \delta^{-3}\left(\mathrm{~d}^{2} / \mathrm{d} \xi^{2}\right)-1$ and the governing equations and boundary conditions 
become

$$
\begin{gathered}
\mathcal{L}^{2} u=T v\left(V_{3}+\delta^{3 / 2} V_{3}^{\prime} \xi+\frac{1}{2} \delta^{3} V^{\prime \prime} \xi^{2}\right), \quad \mathcal{L} v=\frac{1}{2} u\left(V_{3}^{\prime}+\delta^{3 / 2} V^{\prime \prime} \xi\right), \\
u=u^{\prime}=\varepsilon^{2} \delta^{-3} u^{\prime \prime \prime \prime}-2 u^{\prime \prime}=0 \quad \text { at } \quad \xi=0 .
\end{gathered}
$$

We can solve these equations at $\mathcal{O}(1)$ and find that

$$
\begin{array}{r}
u_{0}=\mathcal{H}\left[1-(12)^{1 / 4} \xi / \varepsilon_{0}+e^{-3^{1 / 4} \cos (\pi / 12) \xi / \varepsilon_{0}}\left(\sqrt{3} \sin \left(3^{1 / 4} \sin (\pi / 12) \xi / \varepsilon_{0}\right)\right.\right. \\
\left.\left.-\cos \left(3^{1 / 4} \sin (\pi / 12) \xi / \varepsilon_{0}\right)\right)\right],
\end{array}
$$

where $\mathcal{H}$ is a constant, $\sqrt{1-\omega_{1}}=3^{1 / 4} e^{-\pi \mathrm{i} / 12}, \sqrt{1-\omega_{2}}=3^{1 / 4} e^{\pi \mathrm{i} / 12}$, and we have discarded the exponentially increasing and growing oscillatory solutions at this order as required.

We require that (3.43) matches with the velocity in the outer layer given by the Airy solutions, $\mathcal{F} \operatorname{Ai}(X)$ and (3.41), together with the two oscillating, decaying WKB solutions from (3.31). To achieve this requires $\operatorname{Ai}\left(-\lambda_{1} \lambda_{0}^{-2 / 3}\right)=0$ and $\mathcal{H}=\widetilde{\mathcal{H}} \delta^{1 / 2}$ with $\widetilde{\mathcal{H}}=\mathcal{O}(1) ;$ in particular

$$
\begin{gathered}
\lambda_{1} \lambda_{0}^{-2 / 3}=\frac{\tau_{3}}{3 \tau_{0} \varepsilon_{0}^{2}}\left[-\frac{1}{3 \varepsilon_{0}^{2}}\left(\frac{V^{\prime \prime}}{V_{3}^{\prime}}+\frac{V_{3}^{\prime}}{V_{3}}\right)\right]^{-2 / 3}=2.3381 \\
\mathcal{F}\left(2.3381 \frac{2 \varepsilon_{1}}{3 \varepsilon_{0}}-\frac{\tau_{6}}{3 \varepsilon_{0}^{2} \lambda_{0}^{2 / 3}}\right) \mathrm{Ai}^{\prime}(-2.3381)=\tilde{\mathcal{H}} \\
\mathcal{F} \lambda_{0}^{1 / 3} \mathrm{Ai}^{\prime}(-2.3381)=-(12)^{1 / 4} \tilde{\mathcal{H}} / \varepsilon_{0}
\end{gathered}
$$

Matching the oscillatory, decaying solutions yields

$$
\begin{gathered}
\mathcal{E}_{-}\left(1-\omega_{1}\right)^{-1 / 4}\left(V_{3}^{\prime}\right)^{-1 / 3} \exp \left\{\frac{1}{\varepsilon} \int_{x_{2}}^{0} g_{-1}(s) \mathrm{d} s+\frac{T_{6}}{6 \varepsilon_{0} T_{0}} \int_{x_{2}}^{0} \frac{g_{-1}(s)^{2}-1}{g_{-1}(s)} \mathrm{d} s\right\} \\
=\delta^{1 / 2} \widetilde{\mathcal{H}} e^{4 \pi \mathrm{i} / 3}
\end{gathered}
$$

We now have all the necessary equations. First, observe that the two Taylor number expansions (2.11) must be equivalent. We expand $\tau_{0}, T_{0}$ as series in $\delta$ using $(3.9),(3.34)$ and equate coefficients. At $\mathcal{O}(\delta)$ we infer $\tau_{3}=1.6501$ and then (3.44a) is used to determine $\varepsilon_{0}$ :

$$
\varepsilon_{0}=\left(\frac{\tau_{3}}{7.01432 \tau_{0}}\right)^{3 / 2}\left[-\frac{1}{3 \varepsilon_{0}^{2}}\left(\frac{V^{\prime \prime}}{V_{3}^{\prime}}+\frac{V_{3}^{\prime}}{V_{3}}\right)\right]^{-1}=0.0688+0.1631 \delta+\mathcal{O}\left(\delta^{2}\right) .
$$

From the arguments of $\S 3.2$ we know that $a$ is exponentially close to $-1 / 2$ so that, from (3.12),

$$
\tau_{6}=T_{6}=-\frac{3}{2} \varepsilon_{0} T_{0}\left(\frac{-2 V^{\prime \prime}}{V_{0}}\right)^{1 / 2}=0.2421+0.4082 \delta+\mathcal{O}\left(\delta^{2}\right) .
$$


It follows from (3.44c) and (3.44b) respectively that

$$
\widetilde{\mathcal{H}} / \mathcal{F}=-0.2741+0.0018 \delta+\mathcal{O}\left(\delta^{2}\right), \quad \varepsilon_{1}=-0.0105-0.0134 \delta+\mathcal{O}\left(\delta^{2}\right) .
$$

Our final conditions are given by equation (3.45). Using (3.16), (3.26), (3.32) we find that

$$
\mathcal{E}_{-} \sim 0.7439\left(\frac{1}{2}+a\right) \delta^{3 / 8} \exp \left\{\frac{1}{\varepsilon} \int_{x_{0}}^{x_{2}} g_{-0} \mathrm{~d} s+1.3195 \int_{l_{0}}^{x_{2}} \frac{g_{-0}^{2}-1}{g_{-0}} \mathrm{~d} s\right\} \in \Re .
$$

Equating magnitudes and argument in (3.45) yields

$$
\begin{array}{r}
\frac{1}{2}+a \sim 3.1091 \widetilde{\mathcal{H}} \delta^{1 / 8} \exp \left\{-\Re\left[\frac{1}{\varepsilon} \int_{x_{2}}^{0} g_{-1}(s) \mathrm{d} s+\frac{T_{6}}{6 \varepsilon_{0} T_{0}} \int_{x_{2}}^{0} \frac{g_{-1}(s)^{2}-1}{g_{-1}(s)} \mathrm{d} s\right]\right. \\
\left.-\frac{1}{\varepsilon} \int_{x_{0}}^{x_{2}} g_{-0} \mathrm{~d} s-1.3195 s\right\} . \\
4 \pi / 3=\pi / 24+\Im\left[\frac{1}{\varepsilon} \int_{x_{2}}^{0} g_{-1}(s) \mathrm{d} s+\frac{T_{6}}{6 \varepsilon_{0} T_{0}} \int_{x_{2}}^{0} \frac{g_{-1}(s)^{2}-1}{g_{-1}(s)} \mathrm{d} s\right] \quad \text { modulus } 2 \pi .
\end{array}
$$

Equation (3.50b) will determine the discrete values of $\delta$ at which the solution has the correct phase to satisfy the boundary conditions. As $\delta \rightarrow 0$, it is clear that the right hand side will increase unboundedly. But we are concerned with its value modulus $2 \pi$ and there are likely to be multiple values of $\delta$ for which this expression is satisfied.

\section{Conclusions}

In the preceding sections we have demonstrated the manner in which a consistent solution of the governing equations for the simultaneous onset of exponentially linked Taylor and Dean instabilities might be constructed. Our consideration of the leading order velocity has determined the leading coefficients in the asymptotic expansions of $\varepsilon$ and the Taylor number $T$. Although not all coefficients are resolved it is clear the way in which the solution might be continued to higher orders.

In order to precisely determine the values of $\delta$ for which the phase matching condition (3.50) is satisfied we would require $\varepsilon$ to be determined to $\mathcal{O}\left(\delta^{3}\right)$. The process is extremely involved but once completed would produce an implicit expression to determine $\delta$ (allowing for the dependence of $g_{-1}, T_{0}$ etc. ). Having demonstrated the approach necessary we do not pursue these higher orders. Our main concern is with the implications for the likely existence and nature of these solutions and the possibility of neutral curve crossing at moderate wavenumbers. The values of $\delta$ which satisfy (3.50) will be discrete - sparse for moderate values of $\delta$ but becoming more closely gathered as zero is approached. Therefore we predict that neutral curve crossing will be almost impossible to observe numerically. 
The values of $\beta$ at which numerical experimentation is performed will need to be precise - any deviation from the discrete theoretical values required will result in exponential divergence from the required solution phase.

In making comparisons with previous studies, and in particular that of [4], it must be remembered that our study concentrates upon high wavenumbers. However, as Kachoyan suggests, the asymptotic behaviours observed here might be used as a guide to interpret the behaviour of neutral curves for other values of $\beta$. The study of Kachoyan considered values of the parameter $\beta \simeq 4.45$ and moderate wavenumbers. For these values he was unable to observe crossing of neutral curves and speculated that the stability curves associated with the Taylor and Dean instabilities possessed a kink. Our findings suggest that it is possible that crossing could occur but that the accuracy required in calculations to observe such a phenomenon is not realisable. In practice the onset of both instabilities are unlikely to occur simultaneously.

\section{References}

[1] Drazin, P. \& Reid, W., Hydrodynamic Stability, Cambridge University Press, 1981.

[2] DiPrima, R., The stability of viscous flow between rotating concentric cylinders with a pressure gradient acting around the cylinders, J. Fluid Mech. 6, 462-468, 1959.

[3] Hall, P., Taylor-Görtler vortices in fully developed or boundary-layer flows: linear theory, J. Fluid Mech. 124, 475-494, 1982.

[4] Kachoyan, B., Neutral curve behaviour in Taylor-Dean flow, ZAMP 38, 905-924, 1987.

[5] Chen, F. \& Chang, M., Stability of Taylor-Dean flow between rotating cylinders, J. Fluid Mech. 243, 443-455, 1992.

[6] Laure, P. \& Mutabazi, I., Nonlinear analysis of instability modes in the Taylor-Dean system, Phys. Fluids 6, 3630-3642, 1994.

[7] Abramowitz, M. \& Stegun, I., Handbook of Mathematical Functions, Dover, 1972.

[8] Heading, J., The Stokes phenomenon and certain $n$ th-order differential equations, Proc. Camb. Phil. Soc. 53, 419-441, 1957.

\section{P. Hills}

Department of Mathematical Sciences

University of Exeter

EX4 4QE

U.K.
Andrew P. Bassom

School of Mathematics and Statistics

University of Western Australia

Crawley 6009

Australia

(Received: October 21, 2003; revised: November 11, 2004)

Published Online First: November 4, 2005

\section{(5) To access this journal online: \\ (4P) http://www.birkhauser.ch}

